Scientific Visualization, 2020, volume 12, number 5, pages 13 - 24, DOI: 10.26583/sv.12.5.02

\title{
Analysis of large visualization datasets for thermographic studies in fluid dynamics
}

\author{
I.A. Znamenskaya ${ }^{1}$, A.M. Shagiyanova² ${ }^{2}$ E.Yu. Koroteeva3, M.I. Muratov4, P.A. Ryazanov5
}

Lomonosov Moscow State University

\author{
1 ORCID: oooo-0oo1-6362-9496, znamen@phys.msu.ru \\ 2 ORCID: oooo-0002-2216-4503, shagiyanova@physics.msu.ru \\ 3 ORCID: 0000-0002-1705-5142, koroteeva@physics.msu.ru \\ 4 ORCID: 0000-0002-6545-5829, muratov583@gmail.com \\ 5 ORCID: oooo-0002-2003-6225, pa.ryazanov@physics.msu.ru
}

\begin{abstract}
This paper reports on the visualization of non-stationary thermal fields for two experimental problems with different temporal and spatial scales using high-resolution infrared (IR) thermography. We study: 1. the near-wall region of the impinging non-isothermal liquid jet and 2. the heat fluxes from the shock-tube walls during the passage of the shock wave. These are the high-speed fluid dynamic processes, and their study involves obtaining and analyzing large amounts of visual data.

For the non-isothermal mixing of an impinging water jet, the flow is analyzed in the region near an IR-transparent wall. The thermograms of non-isothermal vortex flow in the near-wall region are presented. The energy spectra of temperature pulsations are computed for various regions of the wall-jet flow. In the gas-dynamic experiment, the thermal response of the shock tube wall to the shock wave propagation is studied. The infrared imaging of surfaces with different thermal conductivity and emissivity is conducted.

The approaches are discussed for optimizing the registration and analysis of large thermographic datasets.
\end{abstract}

Keywords: infrared thermography, post-processing, thermal radiation.

\section{Introduction}

When studying dynamic processes in plasma, gases, liquids and multiphase media with panoramic methods based on modern digital cameras, problems arise due to the presence of large arrays of digital data which carry useful information about the physical processes being studied. With the constant improvement of digital equipment, the amount of information received by the researcher also increases. In each experiment, tens of thousands of new images can be obtained that are of interest to researchers and the scientific community, provided that they are analyzed and interpreted correctly from a physical point of view. This leads to new tasks related to the storing, systematization, processing, analysis, and presentation of the results of a visual experiment.

A large amount of information and the development of technologies for storing and transmitting it has contributed to the introduction of machine learning into the analysis and processing. However, when getting new knowledge about the object of research in unique experimental conditions, the connection of machine learning is still problematic [1]. This is especially true for problems for which a numerical solution is not yet available, and it is difficult to obtain the initial synthesized data necessary for training. In addition, machine learning is still quite resource-intensive for general use and does not always produce better results than classical methods of analyzing visualized data. 
The development of a new generation of thermal imaging technology made it possible to register two-dimensional thermal fields in dynamics with high spatial and temporal resolution. In this paper, we consider some results obtained with medium-wave infrared (IR) shootings using data processing programs.

Thermography is widely used for the study of heat flows from the surfaces of solids $[2,3]$ and in hydrodynamics $[4,5]$. Another application of thermography is the registration of thermal fields on the surface of a liquid [6 - 9]. Water absorbs medium-wave IR radiation in a thin submillimeter layer, this effect allows you to visualize structures on the surface of the liquid. In the literature, there are mainly works devoted to slow flows with small Reynolds numbers or stationary flows. For the study of liquid media, infrared thermography is often used simultaneously with other imaging methods, such as PIV or tomography [10].

In [11], the structure of a free liquid jet falling on a metal plate was analyzed using IR thermography and digital anemometry based on particle images. The combined use of mediumwave and near-infrared thermography [12] is used for the study of liquid films. In these works, thermographic data reflect the properties of the flow at the gas-liquid interface, or the result of the influence of the submerged flow.

Thermal imagers have proven their effectiveness in analyzing the dynamics of complex nonisothermal flows due to the possibility of high-speed shooting - the registration frequency can reach several $\mathrm{kHz}$ depending on the spatial resolution. Modern devices have high sensitivity (up to $20 \mathrm{MK}$ ) [13], which is successfully used for studying heat transfer, in particular, caused by turbulent flow.

Previously, a method for thermography of high-speed flows was proposed [14]. As a result of its application to the study of a number of model jet flows, large experimental data amounts were obtained. The method involves thermal imaging registration through the IR-transparent wall of the reservoir from the boundary layer of the liquid. In this case, the flow movement indicator is a turbulent vortex structure with different temperatures. The quantitative data obtained by the proposed method for turbulent boundary layers of jet flows can be used, in particular, for numerical calculations when verifying the results of numerical simulation of turbulent flows [15].

\section{Thermal imaging registration}

Most currently used thermal imagers operate in the medium-wave (3 - 5 microns) and long wave (8-14 microns) spectral bands. In this work, a FLIR SC7700 thermal imaging camera operating in the medium-wave IR range of 3.7-4.8 microns was used for panoramic registration of dynamic thermal fields. This device has a high temperature sensitivity (NETD) of up to 20 MK. For full-frame mode (640x512 pixels), the maximum shooting frequency is $115 \mathrm{~Hz}$ and with limited resolution is to $400 \mathrm{~Hz}$. The spatial resolution for the described experiments is $0.08-0.15 \mathrm{~mm} /$ pixel.

Increasing the volume of obtained results has led to a new problem - the problem of a rational approach to the registration, processing and storage of visualization data arrays. For the thermal imager shooting with a duration of $t=1 \mathrm{~s}$ at a frequency of $f=295 \mathrm{~Hz}$ and a spatial resolution of $348 \times 344$ pixels takes $60 \mathrm{MB}$. The choice of a short time interval for registration is only possible for the study of stationary flows. When analyzing non-stationary flows, a long-term survey is required to ensure continuous recording of the non-stationary phase of the process. Thermal imaging "film" with a duration of $t=600 \mathrm{~s}$ at $f=100 \mathrm{~Hz}$ and a maximum resolution of 640x512 pixels takes up about $40 \mathrm{~GB}$.

\section{Examples of post-processing of dynamic thermal fields}

This paper demonstrates the use of infrared thermography for the study of two dynamic processes of different types in different time and space ranges: turbulent jet flow of a liquid and the passage of a shock wave in a gas. The study of non-isothermal mixing of a liquid on the surface of an IR-transparent wall allows us to study the hydrodynamic structure of the flow 
based on the obtained data on thermal pulsations in the near-surface region of an impact submerged jet. When a shock wave passes through the channel, a thermal response is registered on the solid wall of the gas-dynamic stand. Both tasks are connected with panoramic registration of the flow field and obtaining, processing, and analyzing a large amount of visual information. Initial processing was performed using FLIR Altair software. Then, in accordance with the given task, approaches for quantitative analysis of data sets were chosen.

\subsection{Wall area of an impact non-isothermal liquid jet}

Most experimental studies on the heat exchange of jets with a solid wall are based on the registration of the thermal field of the wall itself. Measurements in the medium face limitations of the method, such as the finite thickness of the laser knife, or its use near the wall in the presence of large gradients. Infrared thermography allows you to visualize directly the wall layer of turbulent fluid flow through an IR-transparent window.

In this paper experiments were performed for nozzles $\mathrm{D}=1-4 \mathrm{~mm}$, flow rates $v_{\text {jet }}=0.7-10$ $\mathrm{m} / \mathrm{s}$ and Reynolds numbers $R e_{j e t}=5000-35000$, calculated for values at the exit of the nozzle. The distance from the nozzle section to the impact surface varied $H / D=1-6$. Registration was conducted through an IR-transparent window with a thickness of $2 \mathrm{~mm}$ made of calcium fluoride with a transmission capacity of more than $90 \%$.

It is known that in the wall area of the impact jet, three characteristic flow areas can be distinguished: the area of flow inhibition, the wall area of flow and the area of flow separation. Post-processing of thermographic films allows us to identify patterns in the dynamics of parameters in certain flow zones.
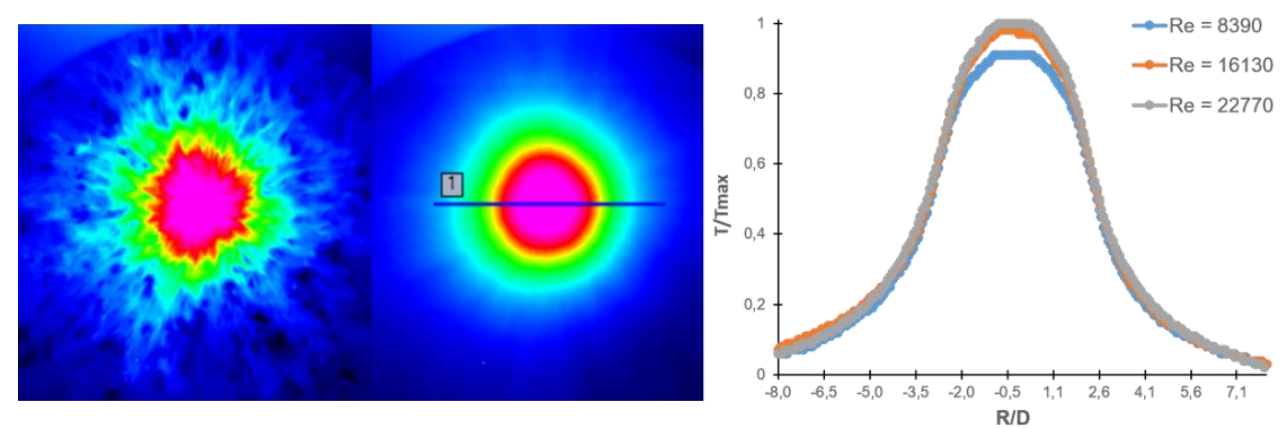

Fig. 1. (from left to right) instantaneous thermogram, averaged thermogram ( $\mathrm{N}=256$ frames) and temperature profiles along line 1 for different flow rates for $H / D=2$.

The stationary component of the non-isothermal flow near the impact surface is visualized using averaged thermograms and temperature profiles. In the area of flow deceleration, the normal velocity to the collision surface reaches zero, the temperature profile reaches a plateau. The shape of the profile depends on the diameter of the nozzle and the distance from the nozzle to the impact surface, and the height depends on the Reynolds number (Fig. 1). In the area of flow deceleration, temperature pulsations are practically not observed (point 1 in Fig. 2).
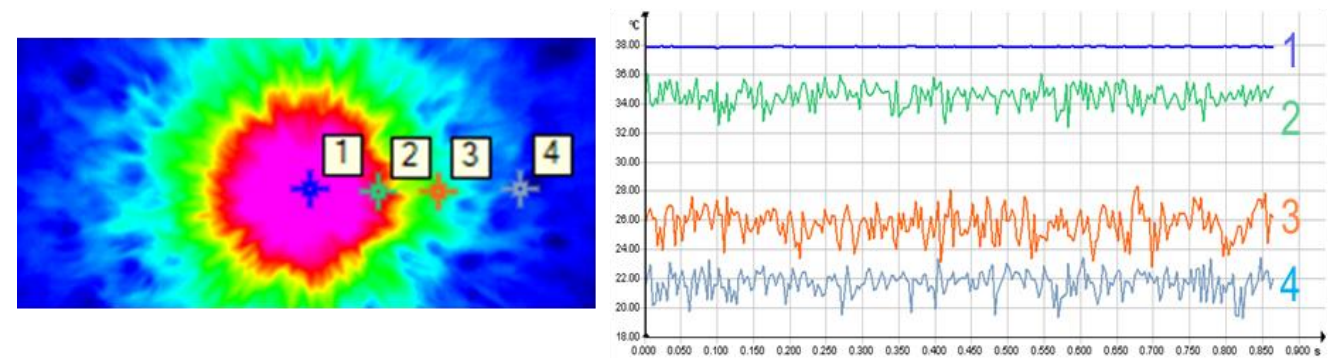

Fig. 2. An example of a thermogram and a time base of the temperature at four different distances from the stagnation point: $R / D=0.2,1.5,3$ and 5 . 
The transition region of the impact jet flow is studied on the basis of registration of nonstationary fields of liquid temperature pulsations. Figure 2 shows an example of a time base of temperature ripples at four different distances $(R)$ from the stagnation point. The observed temperature fluctuations are compared to the mixing process of liquid isothermal elements, since the characteristic times of turbulent mixing are much less than the times of thermal conductivity and natural convection.

The structures in the wall flow are visualized (Fig. 3), obtained by subtracting the average frame: their shape and location in the transition region can give information about the vortex mixing of the wall layer. The size and shape of the structures depend on the parameters of the circular jet flow: The Reynolds number $R e_{j e t}$ and the relative distance from the nozzle slice to the impact surface H/D [19].

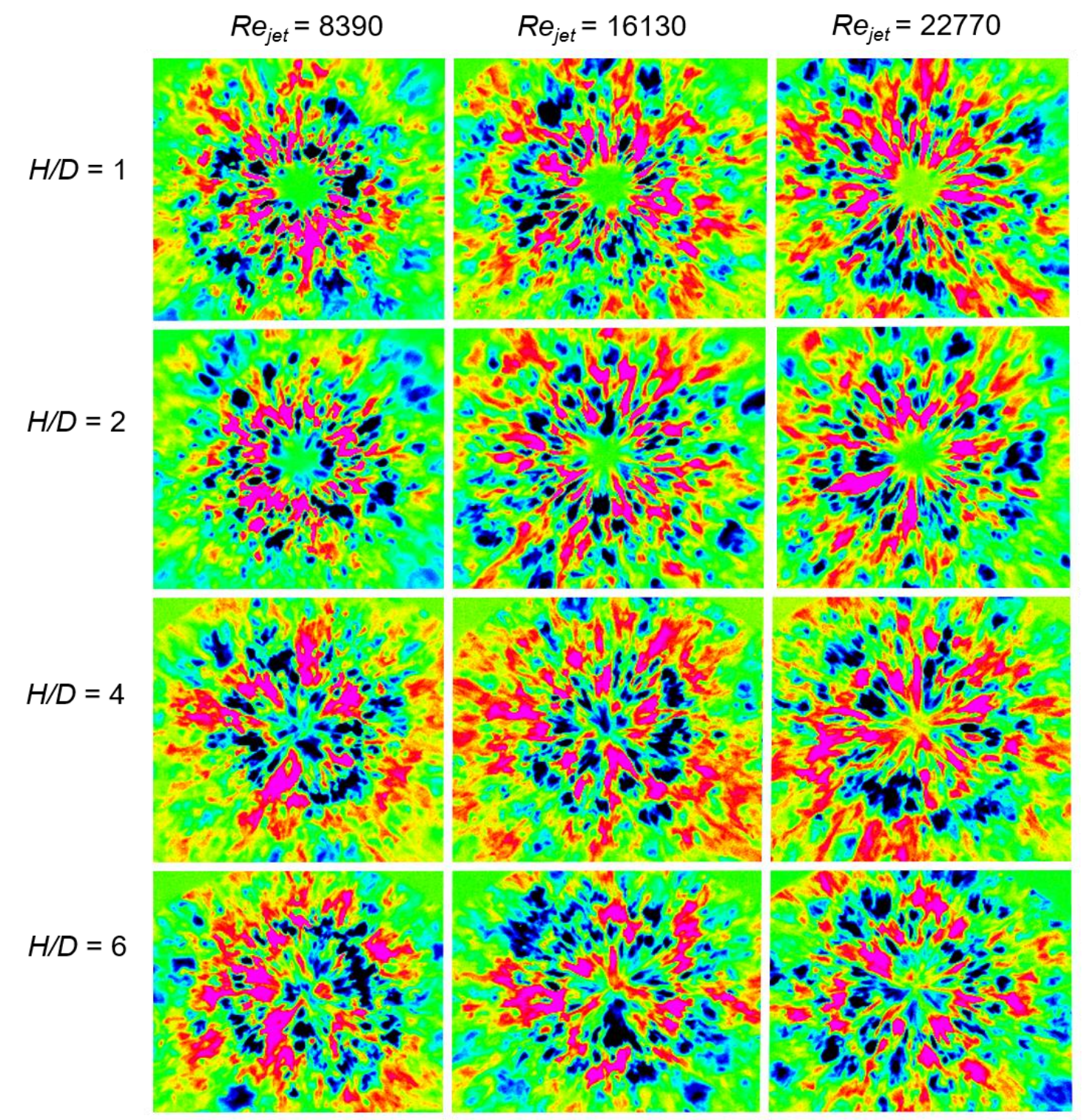

Fig. 3. Instantaneous thermograms of the wall area of the impact jet obtained by subtracting the average frame for $R e_{j e t}=8390,16130,22770$ and $H / D=1,2,4,6$.

The calculation of mean-square pulsations of intensity [20] in the boundary layer of the liquid was also performed, and the values of the radius of maximum pulsations were obtained. Processing of the received arrays of visual data is performed by constructing thermal ripple spectra of the turbulent boundary layer of the liquid. At the selected points of the flow, time scans of temperature pulsations are registered, which are used to construct energy spectra in the frequency range from 1 to $150 \mathrm{~Hz}$. By their shape and comparison with known turbulence 
models, we can speak about the features of flow regions and laminar-turbulent transition. Examples of energy spectra in characteristic areas of the impact jet flow $(D=3 \mathrm{~mm}, H / D=3$, $\left.v_{j e t}=2.4 \mathrm{~m} / \mathrm{s}, R e_{j e t}=7700\right)$ are presented in figure 4 .
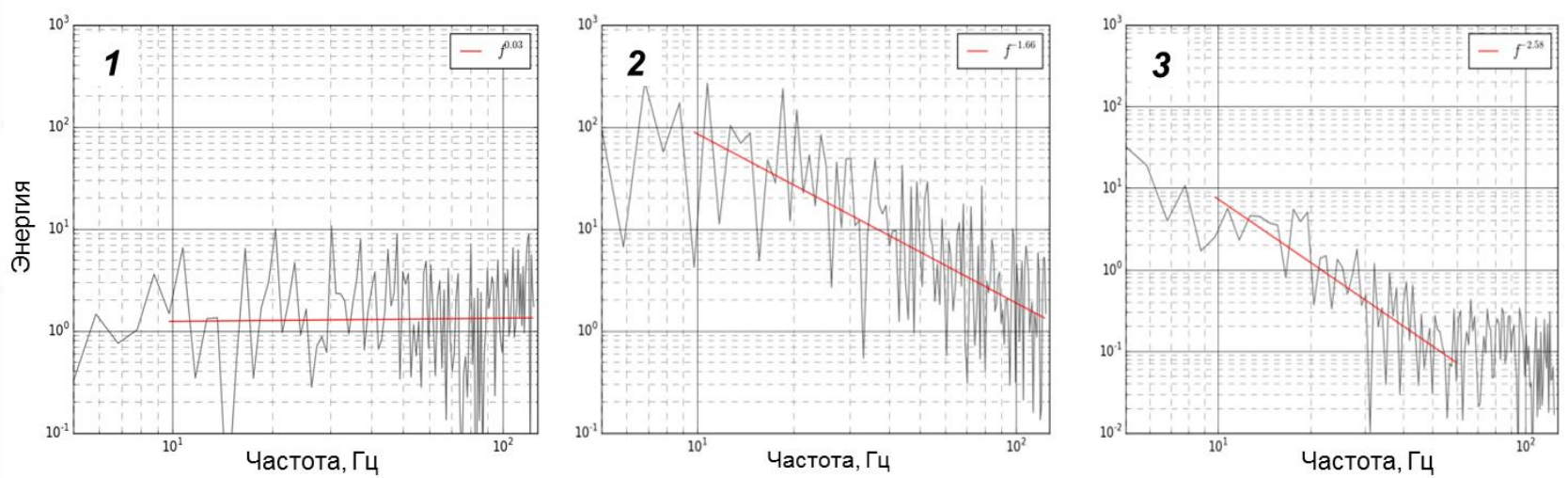

Fig. 4. Typical energy spectra of temperature pulsations at three selected flow points at different distances from the flow stagnation point: $1-R / D \sim 0 ; 2-R / D \sim 4 ; 3-R / D \sim 8$.

The criterion for analyzing energy spectra in this study is their slope calculated using the least squares method. When the flow propagates from the stagnation point in the radial direction, the wall flow is affected by vortex structures of different scales from the outside, changing the turbulent properties of the flow in the visualized area. An example of $3 \mathrm{D}$ visualization of the spectrum slope map is shown in Fig. 5. The color corresponds to the value of slope, and in general the surface has a bell-shaped form. At a distance of 3-6 relative diameters from the impact site, it becomes possible to distinguish a section that is comparable to the classical power law of Kolmogorov "-5/3" and corresponds to the appearance of an inertial interval [21].
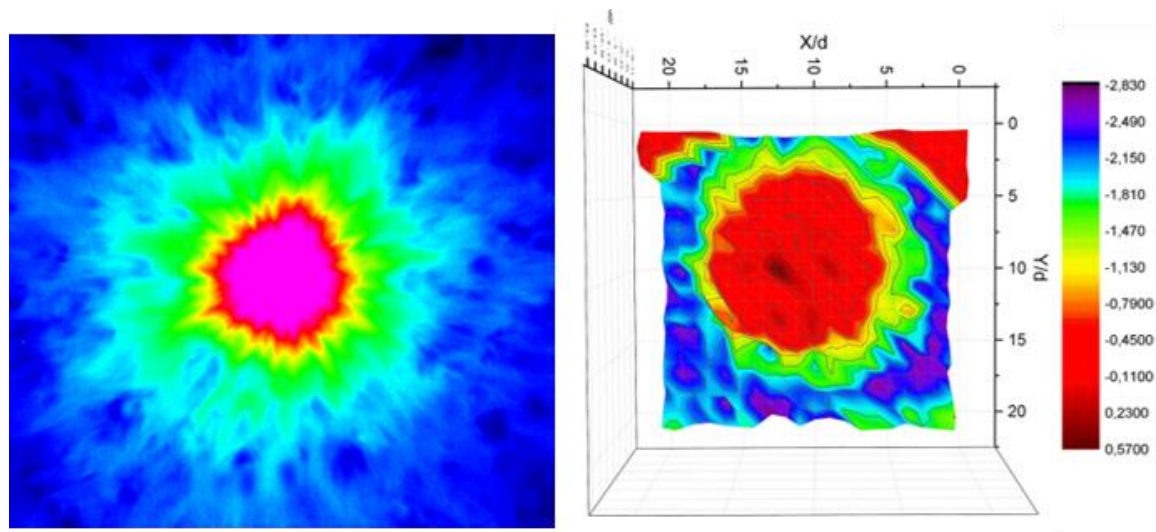

Fig. 5. Power slope map of the near-wall region of the impact submerged jet (on the right) for the $10 * 10 \mathrm{R} / \mathrm{D}$ region (presented on the left).

Another method of post-processing of thermographic data arrays is tracing with heat points, which is considered in [22]. Velocity fields of turbulent non-isothermal fluid flows are obtained using cross-correlation algorithms.

Preliminary analysis of typical process times and spatial scales is necessary to optimize the survey parameters. Choosing the time of registration, frequency of shooting, spatial resolution, as well as the method of processing results in accordance with the task statement and the specific area of flow will lead to a rational use of resources. Based on the results of the analysis of experimental data, the process of post-processing can be represented as a diagram (Fig. 6). 


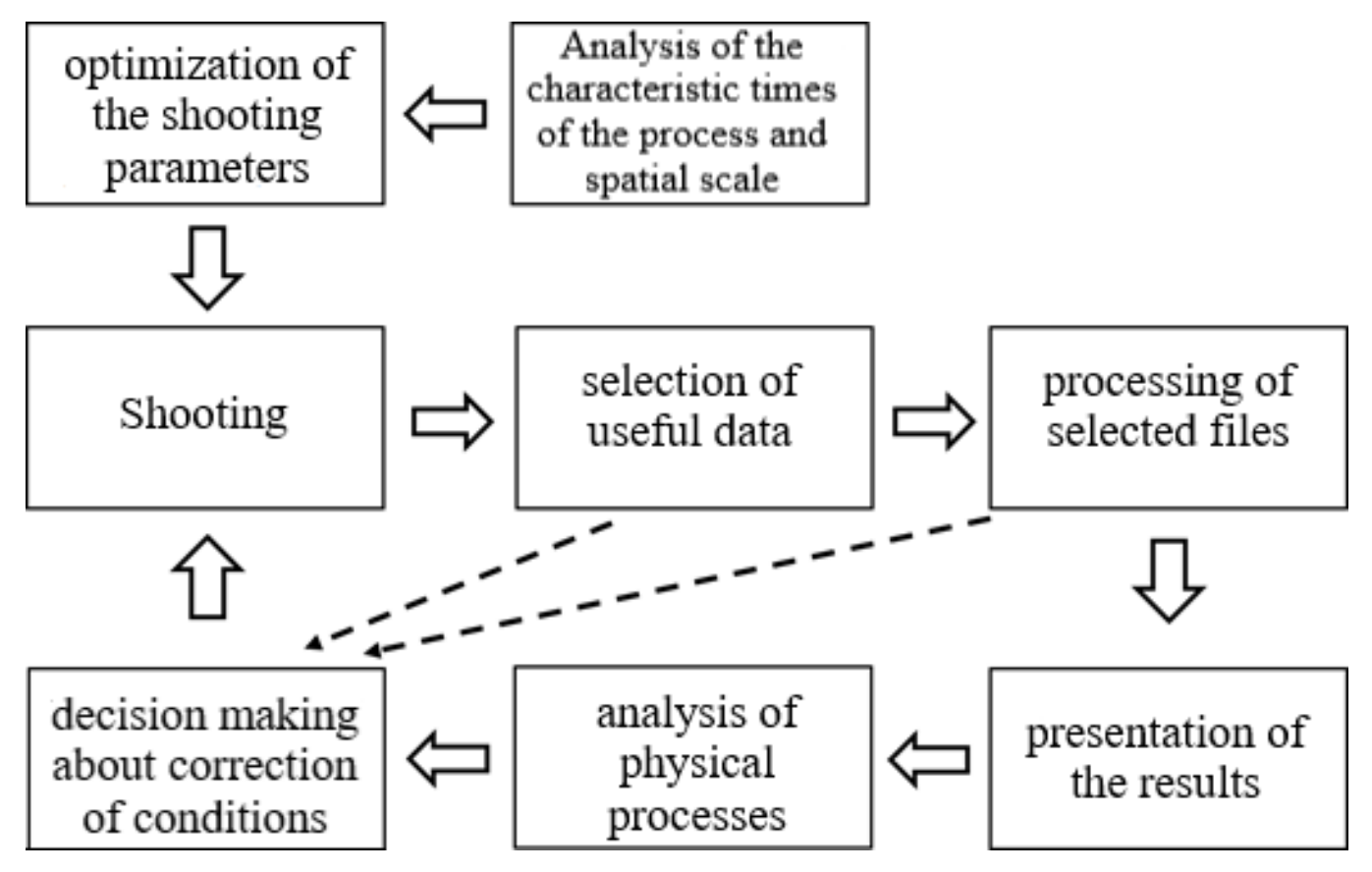

Fig. 6. Scheme of working with experimental data.

\subsection{Shock wave heating of the wall of the channel}

In this problem, thermal imaging was used to study changes in heat flows from the wall of a rectangular channel of a shock tube with a cross-section of $24 \times 48 \mathrm{~mm}^{2}$; the dynamics of heating of the side and end walls of the channel after the passage of a shock wave was obtained. The optical axis of the IR registration was directed perpendicular to the main axis of the shock tube (Fig. 7); radiation was recorded from the regions of the vertical copper wall of the tube $24 \mathrm{~mm}$ high and $2 \mathrm{~mm}$ thick in the section of the low-pressure chamber. The dynamics of heating of the outer wall due to thermal conductivity after the passage of a shock wave in the channel was studied. The flow temperature in the channel behind the shock wave was determined based on the Rankine - Hugoniot conditions [23].

By varying the materials of the diaphragm, as well as the initial pressures in the high and low pressure chambers, a wide range of Mach numbers in the shock tube is achieved. The duration of the passage of the shock-heated flow beside the registration area was 300-400 $\mu \mathrm{s}$, after which the contact surface and the rarefaction wave passed.

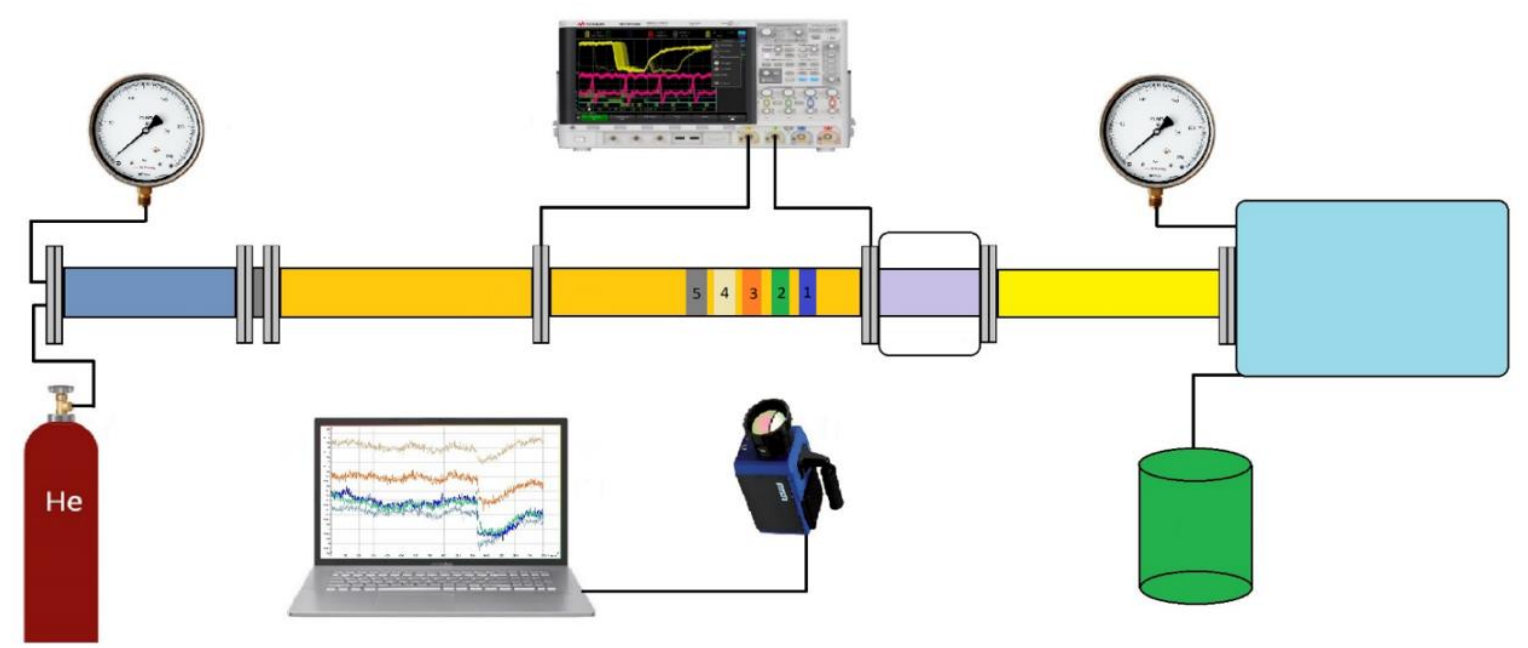

Fig. 7. Experimental installation for a gas-dynamic stand. 
The thermal imager registered radiation from 5 survey areas, which were materials with different thermal conductivity and emissivity deposited on the side surface of the pipe. Coatings: 1 - layer blackened with matte aerosol paint, 2 - black electrical tape, 3 - white masking tape, 4 - a clean surface of the copper pipe and 5 - layer blackened with a marker. Figure 8 shows an instantaneous thermal image of the shock tube wall.

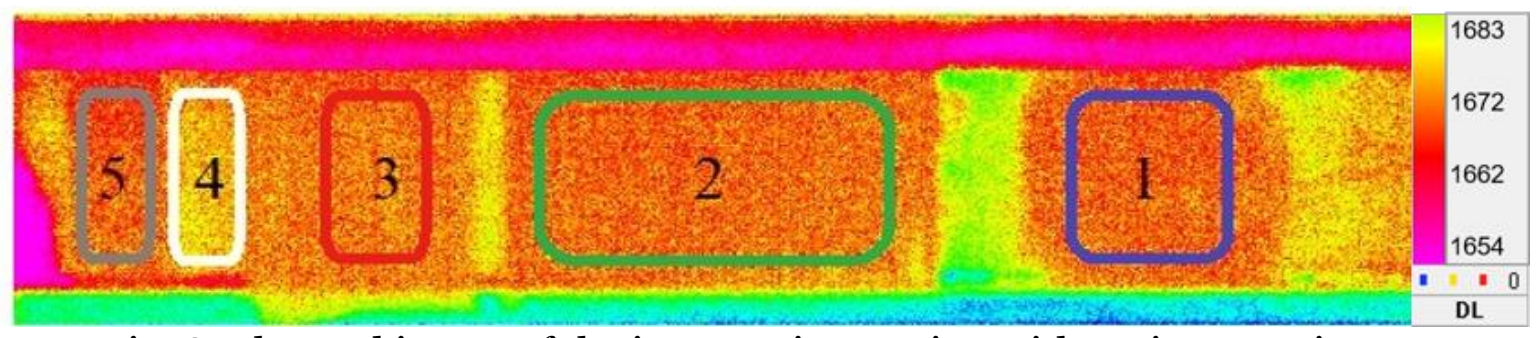

Fig. 8. Thermal image of the impact pipe section with various coatings

For Mach numbers from the range $\mathrm{M}=1,9-3,5$, thermal imaging films with a duration of 5 seconds and a volume of $350 \mathrm{MB}$ were recorded. The shooting frequency was maximum (up to $f=400 \mathrm{~Hz}$ ) due to the small characteristic times of processes inside the channel. Processes caused by the thermal conductivity of the channel walls heated from the inside by the flow were registered. Processing was carried out on the basis of FLIR Altair software. Fig. 9 shows the curves of dependence on the time of temperature change at the points in the centers of the five survey zones.

Due to different coefficients of thermal conductivity of coatings, the time and temperature parameters for survey areas differ (Fig. 9). The fact that the recorded initial temperature of metal and white tape is slightly higher than other materials is explained by the large value of the reflection coefficient of these materials. Also, the thickness of the electrical tape and masking tape does not allow registering a jump in temperature behind the shock wave, while the temperature change on the two black coatings allowed us to draw an analogy with the classical $\mathrm{X}-\mathrm{t}$ diagram for solving equations for the shock tube (Fig. 10).

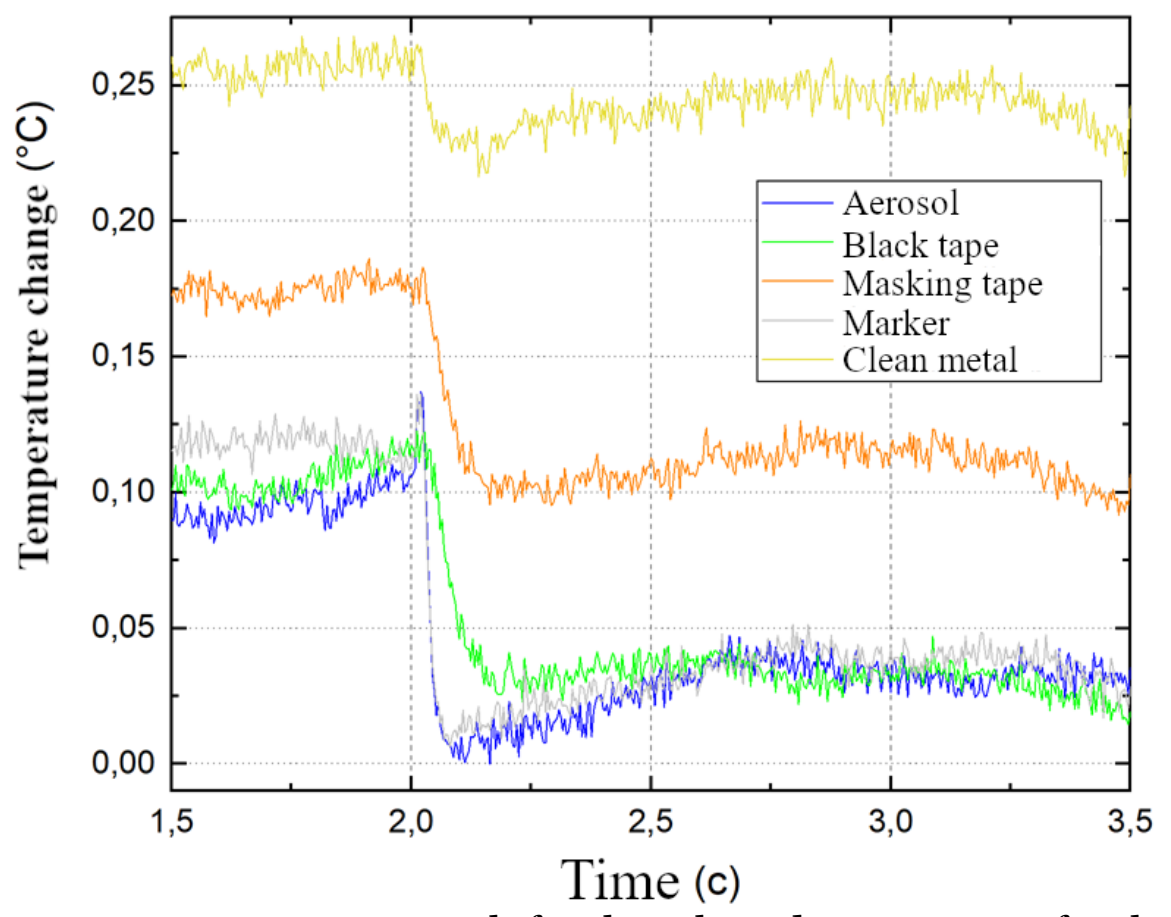

Fig. 9. Temperature measurement result for the selected survey areas for the shock wave Mach number $M=3.5$. 
The abrupt temperature change in the channel corresponds to complex gas-dynamic processes that take place inside the shock tube. The shock wave traveling through the low-pressure chamber heats and compresses the working gas behind it, forming a so-called "plug". The changed parameters of the gas behind the shock wave front, namely, temperature, pressure, and density, are estimated using Rankine-Hugoniot conditions.

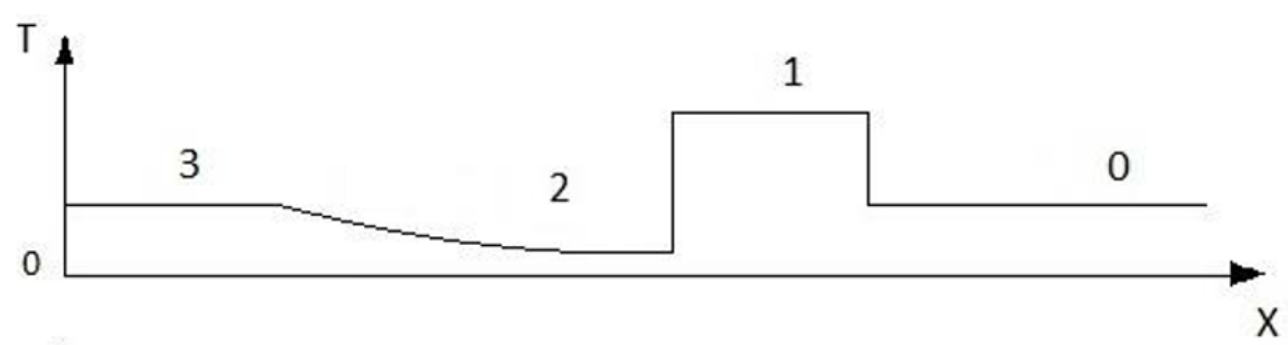

Fig. 10. The temperature profile in the stream channel.

Figure 10 shows the temperature distribution profile in the channel. The heated and compressed satellite stream that goes behind the shock wave front is closed by a contact surface that separates the working and pushing gases. This change in heat flow, observed in all areas of the survey, has some dependence on the Mach number. When analyzing data sets, it was shown that the temperature change is proportional to the increase in the Mach number of the shock wave, starting from $M=2.2$ (Fig. 11). The minimum time recorded by the thermal imager for the rise of heat flow from the wall behind the passing shock wave is about 10 milliseconds for the survey area covered with black aerosol. The change in thermal radiation from the end surface of the pipe, which occurred as a result of double shock compression when reflecting a shock wave, was also studied.

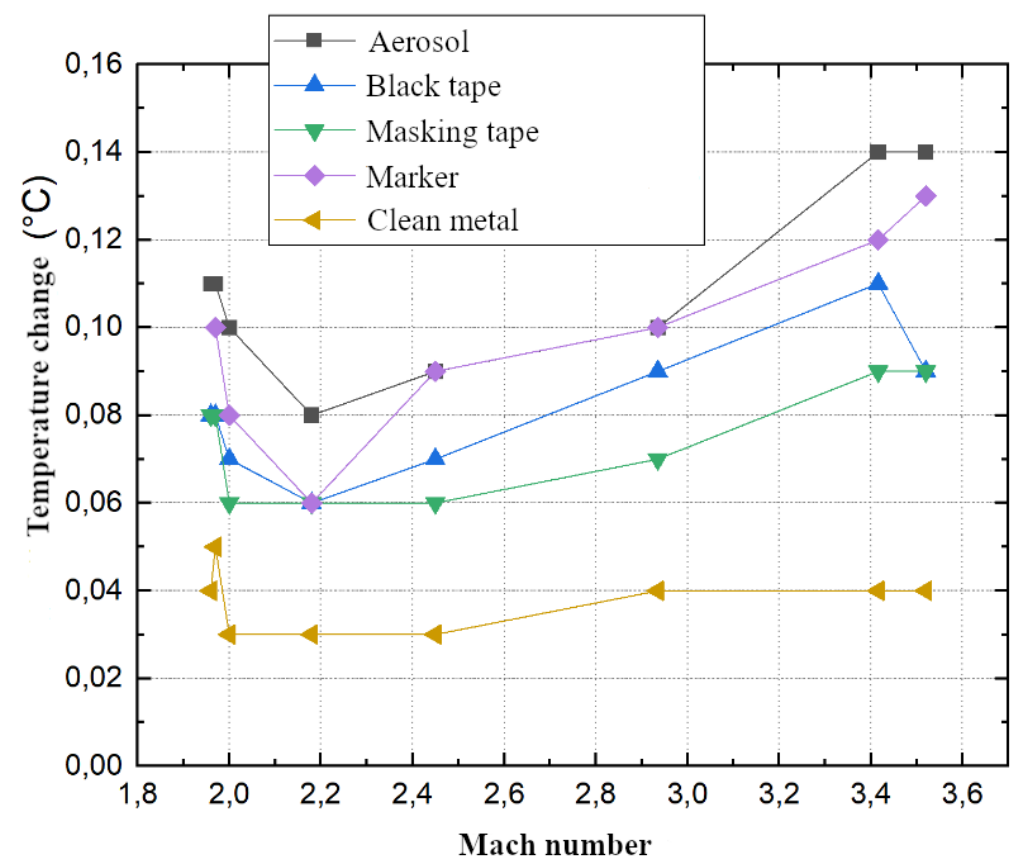

Fig. 11. The dependence of the temperature changes on the Mach number.

\section{Conclusion}

The appearance of a new class of recording infrared technology and new ways to store visual data has led to the accumulation of large amounts of digital experimental data. In this paper, the methods and results of post-processing and analysis of large-volume visual data are pre- 
sented on the example of digital data arrays obtained on two stands - thermophysical and gas-dynamic - for thermographic visualization of high-speed heat flows.

Based on the analysis of the evolution of dynamic thermal fields in the millisecond range of characteristic times, pulsations of turbulent hydrodynamic flow of an impacted submerged liquid jet that occur during non-isothermal mixing near a wall transparent to IR radiation were studied in a wide range of experimental parameters. Both spatial and temporal characteristics of changing thermal fields obtained during panoramic visualization of the jet impact area were analyzed simultaneously; special programs were used to process the data arrays obtained.

For the first time, changes in thermal radiation from the side and end surfaces of the channel during the passage and reflection of the shock wave were registered for the gas-dynamic stand. Measurements were made for several areas of the channel with different thermal conductivity and emissivity of the wall. It is shown that the minimum time recorded by the thermal imager for the increase of heat flow from the outer surface of the wall when the shock wave front passes the survey point is about 10 milliseconds. The quantitative dependences of temperature and time parameters of thermograms on Mach numbers in the range 1.9 - 3.5 (gas temperature behind the shock wave) and the coating material are revealed. The gas dynamic flow in the shock tube is close to one-dimensional; the evolution of parameters was studied with the maximum frequency of shooting, which allowed to resolve processes whose times are limited by the thermal conductivity of the walls.

In studies involving large amounts of panoramic visualization data, additional preliminary analysis of hydrodynamic flows is required in order to minimize the number of measurements and the amount of information received:

- refinement of the survey area parameters (minimize the area and time of registration from the beginning to the end of the process, having previously made an assessment of the useful area);

- optimization of spatial and temporal resolution of registration (rough estimates - by the Nyquist-Shannon sampling theorem);

- $\quad$ ensuring synchronization of the equipment with the beginning and the end of the process.

In this study, the preliminary analysis and use of complexes for processing digital data arrays allowed us to obtain new results, minimize the time of the experiment, and limit the consumption of a limited resource of digital equipment.

The work was supported by an RSF grant 18-19-00672.

\section{References}

1. Cai S., Liang J., Gao Q., Xu C. and Wei R., Particle Image Velocimetry Based on a Deep Learning Motion Estimator // IEEE Transactions on Instrumentation and Measurement. 2019. P. 1-1. (10.1109/TIM.2019.2932649)

2. Vavilov V.P. Infrakrasnaja termografija i teplovoj kontrol' [Infrared thermography and thermal control]. 2nd ed. 2009. P. 544. [In Russian].

3. Leontiev A.I., Kiselev N.A., Burtsev S.A., и др. Experimental investigation of heat transfer and drag on surfaces with spherical dimples // Exp. Therm. Fluid Sci. 79. 2016. P. 74-84. (doi.org/10.1016/j.expthermflusci.2016.06.024)

4. Roux S., Fénot M., Lalizel L.-E. G., Dorigna Brizzi. E. Experimental investigation of the flow and heat transfer of an impinging jet under acoustic excitation // International Journal of Heat and Mass Transfer 54. 2011. P. 3277-3290.

5. Hofmann H. M., Kind M., Martin H. Measurements on steady state heat transfer and flow structure and new correlations for heat and mass transfer in submerged impinging jets // International Journal of Heat and Mass Transfer. 50. 2007. P. 3957-3965. (10.1016/j.ijheatmasstransfer.2007.01.023.) 
6. Ivanitskii G.R., Deev A.A., Khizhnyak E.P. Water surface structure studies using infrared techniques // Uspekhi Fizicheskikh Nauk, 175. N11. 2005. P. 1207 - 1216.

7. Jessup A. T., Phadnis K. R. Measurement of the geometric and kinematic properties of microscale breaking waves from infrared imagery using a PIV algorithm // Measurement Science and Technology, 16(10). 2005. P. 1961-1969. (doi:10.1088/09570233/16/10/011)

8. Judd K.P., Smith G.B., Handler R.A., Sisodia A. The thermal signature of a low Reynolds number submerged turbulent jet impacting a free surface // Physics of Fluids, 20(11). 2008. 115102. (doi:10.1063/1.2981534)

9. Plaksina Y.Y., Pushtaev A.V., Vinnichenko N.A., Uvarov A.V. The effects of small contaminants on the formation of structures during rayleigh-benard-marangoni convection in a planar liquid layer // Moscow University Physics Bulletin. Vol. 73, no. 5. 2018. P. 513519 .

10. Chaugule, V., Narayanaswamy, R., Lucey, A., Narayan, V., Jewkes, J. Particle Image Velocimetry and Infrared Thermography of Turbulent Jet Impingement on an Oscillating Surface // Experimental Thermal and Fluid Science. 98. 2018. P. 576-593. (doi.org/10.1016/j.expthermflusci.2018.06.006)

11. Violato D., Ianiro A., Cardone G., Scarano F. Three-dimensional vortex dynamics and confective heat transfer // International Journal of Heat and Fluid Flow, 37. 2012. P. 2236. (DOI:10.1016/j.ijheatfluidflow.2012.06.003)

12. Dupont J., Mignota G., Paladino D., Prasser H. Mid wave infrared thermography of water films in condensing and evaporating environments// Nuclear Engineering and Design. 2018. N 336. P. 80-89. (doi.org/10.1016/j.nucengdes.2017.06.027)

13. Carlomagno G.M., Cardone G. Infrared thermography for convective heat transfer measurements // Exp Fluids. 49 2010. P. 1187-1218. (doi.org/10.1007/s00348-010-0912-2)

14. Znamenskaya I.A., Koroteeva E.Yu., Novinskaya A.M., Sysoev N.N. Spectral peculiarities of turbulent pulsations of submerged water jets // Technical Physics Letters, 42. № 7 . 2016. P. 686-688.

15. Koroteeva E.Yu. Application of high-speed thermographic visualization for validation of numerical simulations of liquid boundary layer flows. Scientific Visualization 10.2: 2018. P. 112 - 121. (DOI: 10.26583/sv.10.2.09)

16. Nakamura H., Shiibara N., Yamada S. Quantitative measurement of spatio-temporal heat transfer to a turbulent water pipe flow // International Journal of Heat and Fluid Flow. 2017. N 63. P. 46-55. (https://doi.org/10.1016/j.ijheatfluidflow.2016.09.016)

17. Bilskiy, A.V., Lozhkin, Yu.A., Nebuchinov, A.S., The influence of the external periodical excitation on the heat transfer in the impinging jet // Modern Science: Researches, Ideas, Results, Technologies, Iss. №1 (3), 2010. P. 101 - 109.

18. Nebuchinov A. S., Lozhkin Y. A., Bilsky A. V., Markovich D. M. Combination of PIV and PLIF methods to study convective heat transfer in an impinging jet // Experimental Thermal and Fluid Science. V. 80. 2017. P. 139-146. (https://doi.org/10.1016/j.expthermflusci.2016.08.009)

19. Carlomagno G.M., Ianiro A. Thermo-fluid-dynamics of submerged jets impinging at short nozzle-to-plate distance. A review. Experimental Thermal and Fluid Science. 2014. V. 58. P. 15-35.( doi.org/10.1016/j.expthermflusci.2014.06.010)

20.Frik P.G. Turbulence: Approaches and Models // Moscow - Izhevsk: Institute of Computer Science. 2003. P.292.

21. Znamenskaya I.A., Koroteeva E.Yu., Ryazanov P.A., Shagiyanova A.M. Investigations of a laminar-turbulent transition in a water boundary layer based on high-speed thermography // XII Russian Congress on Fundamental Problems of Theoretical and Applied Mechanics. 4 vol. Series Fluid Dynamics. 2. 2019. P. 553-555. [In Russian].

22.Znamenskaya, I.A. Koroteeva E.Yu., Glazyrin F.M. Digital image analysis of liquid and gas-plasma flows based on cross-correlation algorithms. Scientific Visualization 10.4: 2018. P. 111 - 119. (DOI: 10.26583/sv.10.4.08) 
23. Carson L. Runninga, Thomas J. Julianoa, Joseph S. Jewellb, Matthew P. Borgb Hypersonic shock-wave/boundary-layer interactions on a cone/flare // Experimental Thermal and Fluid Science 109. 2019. P.109911.

24.Zeldovich Ya.B., Raiser Yu.P. Physics of shock waves and high-temperature hydrodynamic phenomena // 2013. P. 692. 